\title{
O esvaziamento cervical e o papel da fisioterapia na sua reabilitação
}

\author{
Cervical lymph node dissection and the role of physical therapy in its \\ rehabilitation
}

\author{
Carolina Barreto Mozzini', Rodrigo Costa Schusterer, André Roberto Mozzini ${ }^{3}$
}

\section{Resumo}

O câncer de cabeça e pescoço é de alta incidência no Brasil, ocasionando altas taxas de morbidade e de mortalidade. Com freqüência, há o comprometimento dos linfonodos regionais, localizados na região cervical, e este é um dos indicativos prognósticos importantes nesses pacientes. O esvaziamento cervical é o procedimento cirúrgico mais utilizado, podendo ser realizado de forma radical ou modificado, este último preservando algumas estruturas de importância funcional para o pescoço. O esvaziamento cervical radical ocasiona uma seqüela estética e funcional importante em praticamente todos os pacientes submetidos a esta operação, levando a uma síndrome dolorosa e a perda funcional do membro superior ipsilateral ao procedimento, sendo esta uma complicação importante para o fisioterapeuta. Este estudo tem como objetivo realizar uma revisão de literatura para analisar as complicações deste procedimento cirúrgico e demonstrar a importância da atuação da fisioterapia nestas condições. Através deste estudo, pode-se verificar que os recursos fisioterapêuticos são de grande importância para o tratamento das seqüelas do esvaziamento cervical, mesmo com a escassez de pesquisas na área, os quais têm por objetivo reduzir a dor, minimizar o desconforto, melhorar a funcionalidade da cintura escapular e do membro superior acometido, e conseqüentemente a qualidade de vida dos pacientes.

Palavras-chave: Cirurgia de cabeça e pescoço; Esvaziamento cervical; Fisioterapia; Reabilitação.

${ }^{1}$ Fisioterapeuta.

${ }^{2}$ Fisioterapeuta, mestrando em Ciências Médicas (Universidade Federal do Rio Grande do Sul), professor e supervisor de estágio do Curso de Fisioterapia da Universidade de Passo Fundo.

${ }^{3}$ Médico especialista em Cirurgia de Cabeça e Pescoço, preceptor do Serviço de Cirurgia Geral do Hospital Universitário São Vicente de Paulo de Passo Fundo.

Endereço para correspondência: Carolina Barreto Mozzini. Rua Teixeira Soares 1392, Centro, Passo Fundo/RS CEP: 99010-081. E-mail: carolmozzini@gmail.com 


\section{INTRODUÇÃO}

O câncer é uma doença que, mesmo com os tratamentos disponíveis, provoca muitas seqüelas e apresenta uma alta taxa de mortalidade nos dias atuais. No Brasil, segundo dados do Instituto Nacional de Câncer, as estimativas para o ano de 2006 apontam que ocorrerão aproximadamente 472.050 casos novos de câncer ${ }^{1}$.

$\mathrm{O}$ câncer de cabeça e pescoço representa em média $5 \%$ de todos os tipos de câncer no Brasil e, dependendo do estágio da doença no momento do tratamento, a mortalidade em cinco anos pode chegar a mais de 50\% ${ }^{1}$.

$\mathrm{O}$ tratamento desta modalidade de câncer é complexo e necessita da atuação de uma equipe multidisciplinar, composta por profissionais, dentre os quais se destacam: médicos, enfermeiros, fisioterapeutas, odontólogos, fonoaudiólogos, dentre outros.

Este estudo tem por objetivo realizar uma revisão na literatura sobre a cirurgia de esvaziamento cervical, bem como sobre o papel da fisioterapia na sua reabilitação.

\section{METODOLOGIA}

A revisão de literatura foi realizada a partir da base de dados Bireme (Medline e Lilacs) durante os meses de abril e maio de 2006. Devido à escassez de informaçōes sobre o assunto, buscaram-se inicialmente os artigos dos últimos 40 anos (1966 a 2006), utilizando os seguintes descritores em português: câncer de cabeça e pescoço and esvaziamento cervical and complicações pós-operatórias; fisioterapia and oncologia e, dor pósoperatória and esvaziamento cervical. A busca realizada foi limitada aos artigos publicados em inglês, espanhol, português e alemão. A revisão foi ampliada através de outras fontes como referências citadas nos artigos obtidos, revisōes, metanálises, pesquisas e livros e/ou de referência sobre o tema. O total de 125 artigos foi encontrado nesta busca.

Para a seleção final foram incluídos os artigos que obedeciam aos seguintes critérios: os relacionados ao procedimento de esvaziamento cervical deveriam apresentar descrições básicas sobre a técnica cirúrgica radical e modificada e as complicações funcionais diretas do pós-operatório, fossem essas de uma visão respiratória, ortopédica ou neurológica, que pudessem ser tratadas por meio de fisioterapia; e os relacionados à fisioterapia deveriam incluir as técnicas fisioterapêuticas utilizadas no tratamento de pacientes oncológicos.

Foram excluídos os artigos que descreviam: complicaçõos de exposição, ressecçôes e enxertos da artéria carótida; importância das imagens radiológicas no pós-operatório para identificar tumores recorrentes; estudos sobre tratamento de anormalidades na deglutição; tratamentos psicológicos; aprimoramento e avanços de técnicas cirúrgicas específicas para determinados tumores; complicaçōes eletrocardiográficas pós-operatórias; estudos sobre recorrências dos tumores; estudos sobre a administração medicamentosa; artigos antigos em que se descreviam determinados conceitos considerados ultrapassados por meio de pesquisas atuais, e os estudos que não consideravam os critérios de inclusão. $O$ total de artigos que atendiam a estes critérios foi de 35, porém dez artigos não foram encontrados na sua versão completa. Assim, o número final de artigos utilizados nesta revisão totalizou ${ }^{25}$.

\section{RESULTADOS}

\section{REVISÃO DE LITERATURA}

Os tumores de cabeça e pescoço têm vários locais de origem e tipos histológicos, sendo que mais de $90 \%$ originam-se do epitélio das vias aerodigestivas superiores (cavidade oral, rinofaringe, orofaringe, hipofaringe e laringe $)^{2}$, sendo mais raros nas fossas nasais e seios paranasais. As taxas de incidência de cânceres de vias aerodigestivas superiores em países não-desenvolvidos são superadas somente pelas de câncer do colo uterino, e as taxas de incidência e de mortalidade por câncer de boca em São Paulo e em Porto Alegre são menores, apenas, que as registradas em algumas regiōes da Índia ${ }^{3}$.

A maior parte dos cânceres de vias aerodigestivas superiores é composta por carcinomas espinocelulares, os quais ocorrem com maior freqüência no sexo masculino e nas faixas etárias acima de 50 anos de idade, podendo ocorrer no sexo feminino e em qualquer idade. De acordo com diversos estudos epidemiológicos, os maiores fatores de risco são o consumo de tabaco e de bebidas alcoólicas. Algumas exposiçōes ambientais (profissionais, poluição, ambiente doméstico) e de fatores ligados a deficiências nutricionais também podem aumentar significativamente o risco ${ }^{3}$.

O comprometimento dos linfonodos regionais é um dos indicativos prognósticos mais importantes nos pacientes com câncer de cabeça e pescoço. Os tumores primários sem nódulos metastáticos em linfonodos cervicais têm excelente relação de cura tanto com a cirurgia quanto com a radioterapia. Todavia, a presença de metástases regionais diminui a relação de cura pela metade $^{2}$.

A forma de tratamento para o pescoço, nos últimos 
anos, tem se tornado um dos tópicos de debate mais ativos no campo de oncologia de cabeça e pescoço. Tradicionalmente, a cirurgia é o procedimento mais adotado para os pacientes com evidências clínicas de nódulos metastáticos; porém, atualmente, este procedimento tem sido combinado com a radioterapia. Há controvérsias e ainda permanece sobre investigação a aplicação da quimioterapia para o manejo de doenças do pescoço².

Crile (1906) descreveu o esvaziamento cervical radical (ECR) e Martin (1951) popularizou esta operação, que compreende a remoção de todo o tecido linfático do pescoço, além de estruturas com íntima relação com as cadeias linfáticas cervicais, tais como: músculo esternocleidomastoideo, veia jugular interna $\mathrm{e}$ nervo acessório (XI par craniano). Entretanto, este procedimento é esteticamente deformante e produz uma desordem no ombro homolateral à cirurgia, chamada de "síndrome do ombro doloroso". Na tentativa de diminuir a morbidade deste procedimento, algumas modificações têm sido propostas, as quais incluem preservar as estruturas não-linfáticas, como veia jugular interna (VJI), o músculo esternocleidomastoideo (ETC) e o nervo acessório (NA) ${ }^{4,5}$.

Além disso, existem os esvaziamentos de algumas cadeias específicas, as quais são denominadas ressecções seletivas do pescoço, baseadas com o estágio do tumor primário. O Serviço de Cirurgia de Cabeça e Pescoço do Memorial Sloan-Kettering Cancer Center de Nova York - EUA descreveu a divisão sistematizada dos vasos linfáticos do pescoço, dividindo-os em cinco níveis: I grupo sumentoniano e grupo submandibular; II - grupo jugular superior; III - grupo jugular mediano; IV - grupo jugular inferior; V - grupo triângulo posterior ${ }^{2}$.

Devido às muitas modificações dos esvaziamentos cervicais, várias nomenclaturas foram criadas, causando inúmeras distorções e nomes não-uniformes. Assim, a American Academy of Otolaryngology, Head and Neck Surgery propôs uma classificação para as ressecções de pescoço. O esvaziamento cervical radical é definido como a remoção dos grupos linfáticos de $\mathrm{I}$ a $\mathrm{V}$ incluindo a VJI, NA e ETC. Todos os outros procedimentos são chamados de esvaziamento cervical radical modificado (ECRM), o qual consiste na preservação de uma ou mais estruturas não-linfáticas, as quais são normalmente removidas no ECR. Dentre os ECRM, encontram-se alguns subtipos:

- Tipo I: resseca níveis linfáticos de I a V e preserva o NA;

- Tipo II: resseca níveis linfáticos de I a V e preserva o
NA e ETC;

- Tipo III: resseca níveis linfáticos de I a $\mathrm{V}$ e preserva o NA, ETC e VJI.

Os esvaziamentos cervicais seletivos são os seguintes: - ressecção supra-homo-hióidea do pescoço: resseca níveis linfáticos de I a III e preserva o NA, ETC e VJI; - ressecção lateral (jugular) do pescoço: resseca níveis linfáticos de II a IV e preserva o NA, ETC e VJI; - ressecção anterior do pescoço: resseca níveis linfáticos de I a IV e preserva o NA, ETC e VJI;

- ressecção posterior do pescoço: resseca níveis linfáticos de II a V e preserva o NA, ETC e VJI.

O resultado destas modificações é controverso, mas sabe-se que a função do ombro é mantida quando o NA é preservado ${ }^{2}$. Com este procedimento, as possibilidades de controle do câncer de pescoço permanecem ótimas e o paciente menos freqüentemente experimenta a dor e a disfunção no ombro que resulta da alteração do músculo trapézio ${ }^{4}$. Ao analisar pacientes submetidos a tais procedimentos cirúrgicos, em uma comparação física com 16 dias de pós-operatório através de uma eletroneuromiografia, foi possível verificar que os pacientes submetidos ao ERCM sofrem menos perda funcional e apresentam resultados significativamente melhores do que os submetidos a $\mathrm{ECR}^{6}$.

Conforme a ocorrência de metástase, os esvaziamentos cervicais podem ser classificados segundo a indicação em: de necessidade ou eletivo (ou de princípio). O esvaziamento de necessidade é indicado na presença de um ou mais linfonodos clinicamente metastáticos no pescoço; e o esvaziamento eletivo é indicado na ausência de linfonodo clinicamente positivo, nos tumores malignos em que a experiência mostra ser de alta produção de metástases, pois há a possibilidade de existirem micrometástases que são detectadas clinicamente ${ }^{7}$.

De acordo com a literatura consultada, podem-se evidenciar diversas complicações pós-operatórias nos pacientes com câncer de cabeça e pescoço, sendo que as mais comumente relatadas são dor e inabilidade funcional no ombro; limitação na escala de movimento; alteração e/ou perda de força na musculatura do membro superior e pescoço homolateral à cirurgia, podendo ocasionar depressão do ombro com deslocamento anterior, dependendo das estruturas removidas e da quantidade de fibrose desenvolvida pela cirurgia e pela radioterapia pós-operatória ${ }^{7,8}$ e, ainda, impacto psicossocial ${ }^{2}$. Verificaram-se também complicaçōes incomuns, tais como: neuropatia óptica isquêmica anterior em paciente com fatores de risco pré-existentes ${ }^{9}$; 
fratura de clavícula devido às alterações no movimento de giro do ombro que distorce as forças musculares atuantes sobre a clavícula ${ }^{10}$; tromboembolismo pulmonar em pacientes com doença cardíaca predisponente ${ }^{11}$; paralisia transitória do diafragma ${ }^{12}$; disfagia ${ }^{13}$; fraqueza no lábio inferior após parotidectomias ${ }^{14}$.

Em casos de terapia combinada (cirurgia, quimioterapia e radioterapia) pode ocorrer trauma no nervo auriculotemporal, ramo do nervo mandibular, ocasionando assim a síndrome auriculotemporal, cujos sintomas clássicos são sudorese gustativa, hiperemia e, raramente, dor ${ }^{15}$. Não foram evidenciadas complicações cirúrgicas significativamente diferentes entre pacientes que se submeteram primeiramente à quimioterapia em relação aos que se submeteram primeiramente à cirurgia $^{16}$.

A dor é uma complicação comum, podendo ser aguda - como conseqüência da cirurgia - ou crônica como conseqüência de inabilidade do ombro devido à secção do nervo acessório após esvaziamento cervical. A dor pode ter diversas origens e estudos demonstram que nesses pacientes advém em 35\% dos casos de recorrência do câncer, $30 \%$ de seqüelas do tratamento, $25 \%$ de causas múltiplas, $10 \%$ de causas não-relacionadas. $\mathrm{O}$ tipo mais comum de dor é a mistura de dor nociceptiva e neuropática $(37,5 \%)$, mas a dor nociceptiva isolada é responsável por $32,5 \%$, a miofacial por $13 \%$ e a neuropática por $7,5 \%$; outros tipos mistos de dor também ocorrem em $7,5 \%$ dos casos ${ }^{2,17}$. O caráter e a severidade da dor são influenciados pela localização do câncer, pelo tipo de tratamento e tempo de tratamento pós-operatório ${ }^{2}$. Há correlação entre a dor pósoperatória com o dano no plexo cervical superficial, pois a maior frequiência da dor ocorre em pacientes submetidos ao ECR do que em submetidos ao ECRM tipos I e III ${ }^{18}$.

A qualidade de vida é essencial nos pacientes com câncer. Tem sido demonstrada relação direta entre a presença de dor e a redução da qualidade de vida ${ }^{2,19}$, com impacto significativo no bem-estar geral e na aflição psicossocial $^{2,20}$. Em estudo avaliativo da qualidade de vida após dois anos do procedimento cirúrgico, observou-se a presença de dor forte no ombro e no braço, não demonstrando diferença na sensação de dor entre os indivíduos submetidos ao ECR e aqueles que passaram por procedimentos mais conservadores ${ }^{20}$. Da mesma forma, outros estudos demonstram que em ordem de preferência, os pacientes com câncer de cabeça e pescoço colocam a cura em primeiro lugar, seguido por viver o maior tempo possível, sem dor, ingerindo alimentos e líquidos por via oral e tendo resultados desejados com o tratamento do câncer.

As definições acerca de qualidade de vida são diversas, mas Kaplan et al. limitam a sua avaliação relacionada a três funções: mobilidade, atividade física e atividade social ${ }^{2}$. Indivíduos com preservação do NA apresentam melhores contagens em instrumentos de mensuração de qualidade de vida do que aqueles com NA sacrificado; porém, dentre estes sujeitos, os que apresentam preservação dos linfonodos do nível $\mathrm{V}$ apresentam melhores contagens nos itens comer assim como no domínio da dor, apresentando menor dor no ombro e no pescoço e tiveram menores problemas físicos do que aqueles em que o nível $\mathrm{V}$ foi dissecado ${ }^{19}$.

Em média, os pacientes submetidos ao esvaziamento cervical com a preservação do NA sentem menos dor em seus ombros, sofrem de menos inabilidade funcional, apresentam resultados melhores ao exame físico ${ }^{21,22} \mathrm{e}$ apresentam menores alteraçóes sensoriais ${ }^{23}$ do que os pacientes que têm o NA sacrificado. Em estudo avaliativo da força muscular do ombro no pós-operatório, utilizando um dinamômetro, foram comparados dois grupos de pacientes: um com preservação e outro com separação do NA, e verificou-se que o grupo com preservação de tal nervo apresentava perda de força significativamente menor do que o grupo com separação do $\mathrm{NA}^{24}$. Pesquisas também apontam que os pacientes submetidos a completo tratamento de radioterapia no pescoço sem o esvaziamento cervical apresentaram pouca dor, infreqüente e insignificativa desordem funcional e força normal ao exame físico ${ }^{21}$.

Os pacientes com câncer apresentam freqüentemente limitaçōes funcionais, resultantes da fadiga, descondicionamento, dor ou metástases musculoesqueléticas e nervosas ${ }^{25}$. Um dos profissionais que trabalha de forma direta com o paciente oncológico é o fisioterapeuta, durante sua reabilitação e também no tratamento paliativo, cujo sintoma mais freqüente é a dor, a qual provoca sofrimento ${ }^{26}$. Em pacientes submetidos ao esvaziamento cervical, devido a todas as complicações já expostas, torna-se de fundamental importância a integração de intervenção farmacológica, tratamento da depressão e tratamento fisioterapêutico ${ }^{8}$.

Os recursos fisioterapêuticos e exercícios podem auxiliar na redução da dor, melhorar a qualidade de vida, minimizar o desconforto, bem como aumentar as funçóes, como a do braço e a do ombro .

A cinesioterapia é fundamental em todo o processo de reabilitação, podendo ser iniciada com a movimentação passiva no pós-operatório imediato $\left(3^{\circ}\right.$ 
dia) e a movimentação ativa e exercícios de fortalecimento da musculatura podem ser iniciados assim que os drenos forem retirados. Este cuidado, de uma a duas vezes ao dia, é importante não somente do ponto do vista fisiológico, mas também do ponto de vista emocional. A fisioterapia deve ser realizada de modo a impedir a redução da amplitude de movimento, fazendo com que os pacientes tenham diminuição das deformidades, motivando-os assim a continuar o tratamento fisioterapêutico ${ }^{6}$.

Comumente, na presença de dor oncológica, os pacientes reduzem a sua movimentação e sua atividade física como um todo, ocasionando um comprometimento gradual do condicionamento físico, da força muscular, da flexibilidade e da capacidade aeróbica, fatores esses que com freqüência levam o paciente à chamada síndrome da imobilização, a qual compromete a coordenação motora, reduz a amplitude de movimento articular e acarreta retrações tendíneas. Assim como no pós-operatório, a cinesioterapia nesta etapa se torna um recurso de grande valia, visto que auxilia na restauração e na melhora do desempenho funcional dos segmentos acometidos, desenvolvendo propriocepção, o movimento, a força e o trofismo muscular, prevenindo a imobilidade no leito e devolvendo a amplitude de movimento articular ${ }^{26}$.

As técnicas de terapia manuais podem ser utilizadas como um recurso para a complementação do alívio da dor, pois produz estimulação mecânica dos tecidos, através da aplicação rítmica de pressão e estiramento, reduzindo assim a tensão muscular, melhorando a circulação tecidual e auxiliando na redução da ansiedade do paciente, proporcionando uma melhora na qualidade do sono e da qualidade de vida ${ }^{26,27}$.

A termoterapia, através do calor superficial, é usada freqüentemente para reduzir o desconforto e promover o relaxamento muscular através da interferência no ciclo dor-espasmo-dor devido à redução da atividade das fibras aferentes do fuso muscular (tipo II) e o aumento da atividade das vias aferentes dos órgãos tendinosos de golgi, bem como pela remoção de produtos do metabolismo e mediadores químicos responsáveis pela indução da dor. Porém, a aplicação deste recurso é contra-indicada em áreas pós-cirúrgicas, devido à perda de sensibilidade no local, ou diretamente sobre as áreas do tumor maligno, visto que provoca vasodilatação, aumentando assim a irrigação sangüínea no local e apresentando risco de disseminação de células tumorais por via sangüínea e/ou linfática ${ }^{6,26}$. Da mesma forma, todas as formas de calor profundo também estão contra- indicadas ${ }^{26}$.

Não foram encontrados estudos que comprovem a eficácia da crioterapia para a dor oncológica, mas a sua aplicação pode ser útil para as dores musculoesqueléticas, através de bolsas ou imersão em água gelada, duas a três vezes ao dia, durante 15 a 20 minutos $^{28}$. Porém, deve ser evitada a sua utilização em regiōes onde não há integridade sensorial, comprometimento arterial periférico, em casos de intolerância ao frio ou alergia e onde o tumor compressivo pode ser o causador da redução da circulação local e em áreas de tratamento de radioterapia ${ }^{26}$.

Dentre as intervenções fisioterapêuticas para a dor, a eletroterapia traz rápidos resultados; todavia, o alívio é variável entre os pacientes. No entanto, não é possível tratar a dor oncológica apenas com a utilização de corrente analgésica, mas, de acordo com estudos, podese diminuir de maneira significativa o uso de analgésicos e seus efeitos colaterais ${ }^{29}$.

Outro estudo, utilizando TENS (Estimulação Elétrica Transcutânea), verificou que o uso desta corrente diminui em até $47 \%$ o uso de morfina comparado com o TENSplacebo (não ligada), ocorrendo também diminuição da percepção da dor através da Escala Análoga Visual, associada à incidência de náuseas e prurido de forma significativa ${ }^{30}$. O que determina a percepção individual de um estímulo doloroso é a "abertura" ou "fechamento" da comporta a esses estímulos. Como a condução elétrica da TENS ocorre através das grandes fibras mielínicas, as quais são altamente sensíveis à estimulação elétrica e de condução rápida, levando rapidamente a mensagem até a medula espinhal, a TENS pode propiciar um mecanismo que mantenha o portão fechado a esses estímulos. O mecanismo pelo qual é impedido esse trânsito ocorre através das fibras nociceptivas (condutoras de dor). Quanto mais próximo da área afetada se puder aplicar a TENS, maior será a chance de inibir os estímulos nocivos ${ }^{31}$. Esse processo de redução ou minimização da transmissão da dor é conhecido como neuromodulação. Portanto, esta corrente pode ser utilizada com segurança em pacientes oncológicos, todavia deverá ser aplicada onde a sensibilidade tátil estiver preservada e a pele estiver íntegra ${ }^{26}$.

No caso da incomum ocorrência de paralisia transitória do diafragma, que ocorre devido a uma diferença parcial na pressão do oxigênio arterial alveolar, faz-se necessário o uso de oxigênio complementar para prevenir pneumotórax, paralisia do diafragma e gases sangüíneos arteriais no pós-operatório imediato ${ }^{12}$. 
O prognóstico dos esvaziamentos cervicais depende do tipo histológico do tumor, da localização e do tratamento executado. De forma global, a sobrevida de cinco anos é de 41,49\% de acordo com estudos; nos casos onde não se encontrou linfonodo positivo é de $64,85 \%$; onde há um positivo é de $44,44 \%$; com dois positivos $32,47 \%$ e com três positivos a sobrevida cai para $7,81 \%^{14}$. Assim, percebe-se que quanto mais precoce for o diagnóstico e o tratamento, melhor será resultado e o prognóstico dos pacientes.

\section{CONCLUSÕES}

A cirurgia de esvaziamento cervical é o principal recurso para o tratamento do câncer de cabeça e pescoço com metástases cervicais, podendo ser radical ou modificada; todavia, freqüentemente, resulta em seqüelas que, na maioria das vezes, são visíveis e deformantes, associadas a desordens funcionais principalmente no ombro homolateral ao procedimento, o qual apresenta-se com síndrome dolorosa, alteração na amplitude de movimento e na força muscular.

A fisioterapia, através de seus recursos básicos, pode ser amplamente utilizada para o tratamento das complicaçôes, trazendo grande melhora na qualidade de vida destes pacientes, porém, raros são os estudos documentados na área, fato este que demonstra a necessidade de um maior número de pesquisas sobre a atuação da fisioterapia no câncer de cabeça e pescoço.

\section{REFERÊNCIAS}

1. Instituto Nacional de Câncer (INCA/MS). [homepage na internet]. Estimativa de Câncer 2006. Disponível em: $<$ http://www.inca.gov.br/estimativa/2006>

2. Andersen PE, Saffold S. Management of cervical metastasis. In: Shah J. Cancer of the head and neck. London: BC Decker Inc; 2001:275-87.

3. Angelis EC, Furia CLB, Mourão LF, Kowalski LP. A atuação da fonoaudiologia no câncer de cabeça e pescoço. São Paulo: Lovise; 2000.

4. Jesse RH, Ballantyne AJ, Larson D. Radical or modified neck dissection: A therapeutic dilemma. Am J Surg. 1978;136(4):516-19.

5. Smullen JL, Lejeune FE. Complications of neck dissection. J La State Med Soc. 1999;151(11):544-47.

6. Sobol S, Jensen C, Sawyer W, Costilo P, Thong N. Objective comparison of physical dysfunction after neck dissection. Am J Surg. 1985;150(4):503-509.

7. Sobrinho JA. Esvaziamentos cervicais. In: Brandão LG, Ferraz AR. Cirurgia de cabeça e pescoço. vol 1. São Paulo:
Roca; 1989:189-93.

8. Sigler BA. Nursing care for head and neck tumor patients. In: Thawley SE, Panje WR, Batsakik JG, Lindberg RD. Comprehensive management of head and neck tumors. vol 1. 2nd ed. Philadelphia: Saunders; 1999:59-78.

9. Wilson JF, Freeman SB, Breene DP. Anterior ischemic optic neuropathy causing blindness in the head and neck surgery patient. Arch Otolaryngol Head Neck Surg. 1991;117(11):1304-306.

10.Lörz M, Bettinger R, Desloovere C, Leppek R. Klavikulafrakturen nach radikaler Neck dissection [Clavicular fractures after radical neck dissection]. HNO. 1991;39(4):147-50.

11. Spires JR, Byers RM, Sanchez ED. Pulmonary thromboembolism after head and neck surgery. South Med J. 1989;82(9):1111-115.

12. Moorthy SS, Gibbs PS, Losasso AM, Lingeman RE. Transient paralysis of the diaphragm following radical neck surgery. Laryngoscope. 1983;93(5):642-44.

13. McConnel FM, O'Connor A. Dysphagia secondary to head and neck cancer surgery. Acta Otorhinolaryngol Belg. 1994;48(2):165-70.

14. O'Brien CJ, Petersen-Schafer K, Papadopoulos T, Malka V. Evaluation of 107 therapeutic and elective parotidectomies for cutaneous melanoma. Am J Surg. 1994;168(5):400-403.

15. Hanowell S, Lees DE, MacNamara T. Auriculotemporal syndrome after combined modality therapy for cancer. South Med J.1979;72(9):1116-117.

16. Proctor E, Robbins KT, Vieira F, Hanchett C, Sommes G. Postoperative complications after chemoradiation for advanced head and neck cancer. Head Neck. 2004;26(3):272-77.

17. Sist T, Miner M, Lema M. Characteristics of postradical neck pain syndrome: a report of 25 cases. J Pain Symptom Manage. 1999;18(2):95-102.

18. Agha-Mir-Salim P, Schulte-Mattler W, Funk U, Lautenschläger $\mathrm{C}$, Bloching $\mathrm{M}$, Berghaus A. Entstehung von Schulterschmerzen nach [quot ]neck dissection [Origin of shoulder pain after [quot] neck dissection [quot]. Importance of the cervical plexus. HNO. 2002;50(6):544-52.

19. Terrell JE, Welsh DE, Bradford CR, Chepeha DB, Esclamado RM, Hogikyan ND, et al. Pain, quality of life, and spinal accessory nerve status after neck dissection. Laryngoscope. 2000;110(4):620-26.

20. Chaplin JM, Morton RP. A prospective, longitudinal study of pain in head and neck cancer patients. Head Neck. 1999;21(6):531-37.

21. Short SO, Kaplan JN, Laramore GE, Cummings CW. Shoulder pain and function after neck dissection with or without preservation of the spinal accessory nerve. Am J Surg. 1984;148 (4):478-82.

22. Sesterhenn K, Zilkens J.Zur schmerzhaften schultersteife nach radical neck dissection [The painflu stiff shoulder following 
radical neck dissection]. HNO. 1977;25(7):232-35.

23. Brown H, Burns S, Kaiser CW. The spinal accessory nerve plexus, the trapezius muscle, and shoulder stabilization after radical neck cancer surgery. Ann Surg. 1988;208(5):654-61.

24. Berghaus A, Holtmann S, von Scheel J, Tausch-Treml R, Herter M. Zur frage der schonung des nervus accessorius bei der neck dissection [Preserving the accessory nerve in neck dissection]. HNO. 1988;36(2):68-73.

25. Schmidt KD. Cancer rehabilitation services in a tertiary care center. Cancer. 2001;92(4 suppl):1053-1054.

26. Sampaio LR, Moura CV, Resende MA. Recursos fisioterapêuticos no controle da dor oncológica: revisão de literatura. Rev Bras Cancerol. 2005;51(4):339-46.

27. Pimenta CAM. Dor oncológica: bases para avaliação e tratamento. O Mundo Saúde. 2003;27(1):98-110.

28. Instituto Nacional de Câncer (INCA/MS). Cuidados paliativos oncológicos: controle da dor. Rio de Janeiro: INCA; 2001.

29. International Association for Hospice and Palliative Care. Manual of palliative care. IAHPC; 2003 [cited 2003 Nov 26]. Available from: <http://www.hospicecare.com>

30. Hamza MA, White PF, Ahmed HE, Ghoname EA. Effect of the frequency do transcutaneous eletrical nerve stimulation on the postoperative opioid analgesic requirement and recovery profile. Anesthesiology. 1999;91(5):1232-238.

31. Frampton V. Estimulação nervosa elétrica transcutânea (TENS). In: Kitchen S, Bazin S. Eletroterapia de Clayton. 10a ed. São Paulo: Manole; 1998:276-94.

\section{Abstract}

Head and neck cancer shows a high incidence in Brazil, with high morbidity and mortality rates. It frequently involves regional lymph nodes located in the cervical region, and this is one of the most important prognostic indicators. Cervical lymph node dissection is the most common surgical procedure in such cases and can be radical or modified, in the latter case preserving some functionally important structures for the neck. Radical cervical lymph node dissection leaves major aesthetic and functional sequelae in virtually all patients submitted to this operation, causing a painful syndrome and functional impairment of the ipsilateral upper limb. This complication is thus highly relevant for the physical therapist. The current study conducted a literature review to analyze the complications of this surgical procedure and demonstrate the importance of physical therapy in such cases. Despite the relatively limited body of research in the area, the literature review highlighted the importance of physical therapy for treating sequelae of cervical lymph node dissection, aimed at reducing pain, relieving discomfort, and improving the functional capacity of the shoulder and upper limb and consequently the patient's quality of life. Key words: Head and neck surgery; Cervical lymph node dissection; Physical therapy; Rehabilitation. 